\title{
Unusual acne conglobata case mimicking cervicofacial actinomycosis: A case report with literature review
}

\author{
Magdalena Piotrkowicz¹, Tomasz Wasyłyszyn², Katarzyna Borowska ${ }^{3}$
}

${ }^{1}$ Depatrment of Internal Medicine with Cardiology Unit, District Hospital in Pultusk, Pultusk, Poland, ${ }^{2}$ Department of
Dermatology, Military Institute of Medicine in Warsaw, Warsaw, Poland, ${ }^{3}$ Department of Histology and Embryology with
Experimental Cytology Unit, Medical University of Lublin, Lublin, Poland

Corresponding author: Prof. Katarzyna Borowska, E-mail: k_borowska@wp.pl

\begin{abstract}
Acne vulgaris is one of the most common skin diseases and one of the most frequent diagnoses for patients who visit a dermatologist. The present article describes one of the most severe kinds of acne - acne conglobata, which in a presence of deep inflammation and concomitant general symptoms, is often described as acne fulminans. The authors discuss about case requiring differentiation of acne conglobata and cervicofacial actinomycosis.
\end{abstract}

Key words: Acne vulgaris; Acne conglobata; Actinomycosis

\section{INTRODUCTION}

Actinomycosis is a bacterial infection caused by Gram positive, anaerobic or microaerophilic bacilli, Actinomyces spp. These are higher prokaryotic bacteria belonging to the family Actinomyceataceae. The most frequent pathogen species encountered is Actinomyces israelii [1] but many different species have been described and are associated with pathogenic conditions specific to anatomical sites [2,3]. Based on the site affected, actinomycosis is clinically classified into cervicofacial, pulmonothoracic and abdominocervical forms, of which the most common form is cervicofacial. Cervicofacial actinomycotic lesions usually present as single or multiple cysts and abscesses or indurated lumps with fistulation and a discharge of sulfur granules [4]. While acne vulgaris is a very common disease, the cases of actinomycosis are relatively rare.

\section{CASE REPORT}

A 16-year-old patient reported to the clinic with skin lesions involving the face, mainly at the mandibular area. These were in form of pustules healing as scabs and eventually leaving scars in the upper part of the cheeks. In the lower parts of the face there were several cyst tumors the size of hazelnuts; inflamed and painful, palpably soft covered with shining red skin. One of the cysts broke during the examination revealing inside the presence of discharge containing yellow grains of a soft substance (Fig. 1). These were very similar to so called "sulfur grains" found in actinomycosis. The disease lasted for at least four years, but during final year, the cysts appeared to introduce a serious discomfort to the patient as besides the increasing pain, he reported an occasional fever. The patient was treated with numerous oral antibiotics, including tetracycline, limecycline, azitromycine and clindamycine. Besides topical treatment with antibiotic solutions, and benzoyl peroxide was performed. No improvement was noticed. While the clinical picture obviously suggested acne, authors hypothesised that there might also be an actinomycosis as a complication or as a separate phenomenon. For this reason, a microscopic examination of the "sulfur grain" obtained during examination, stained with methylene blue was performed at the first visit (Fig. 2). It revealed numerous segmented neutrophils

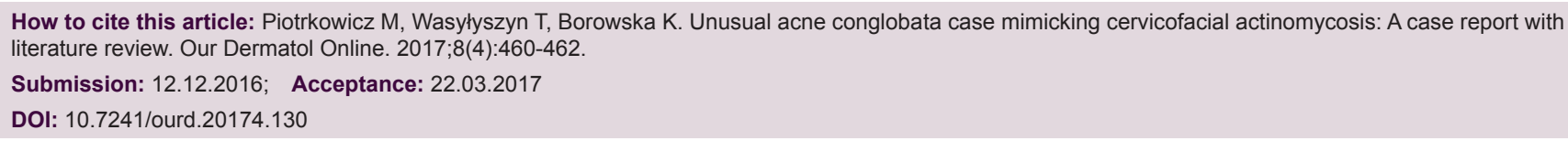




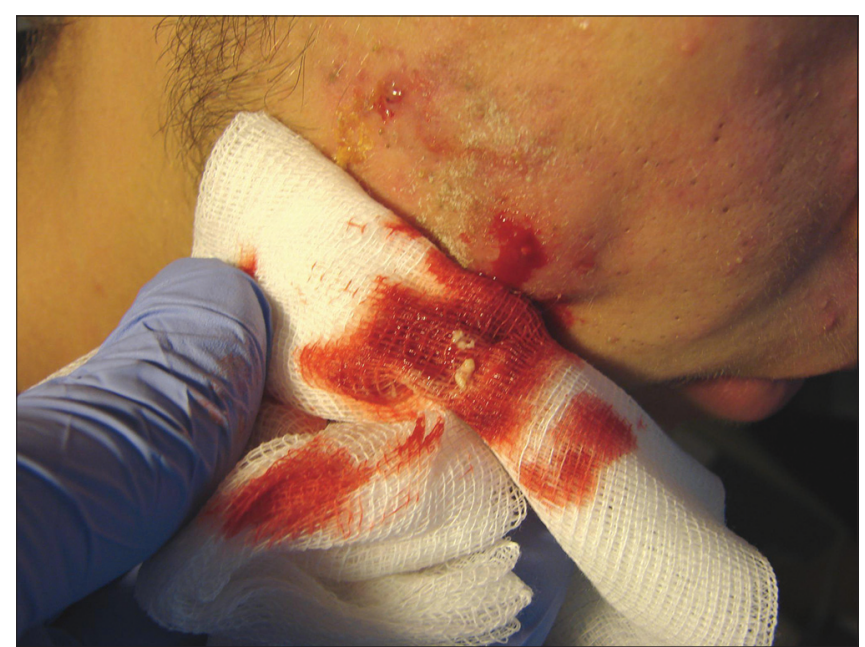

Figure 1: Discharge containing grains of the yellow substance. This phenomenon is very similar to so called "sulfur grains" found in actinomycosis.

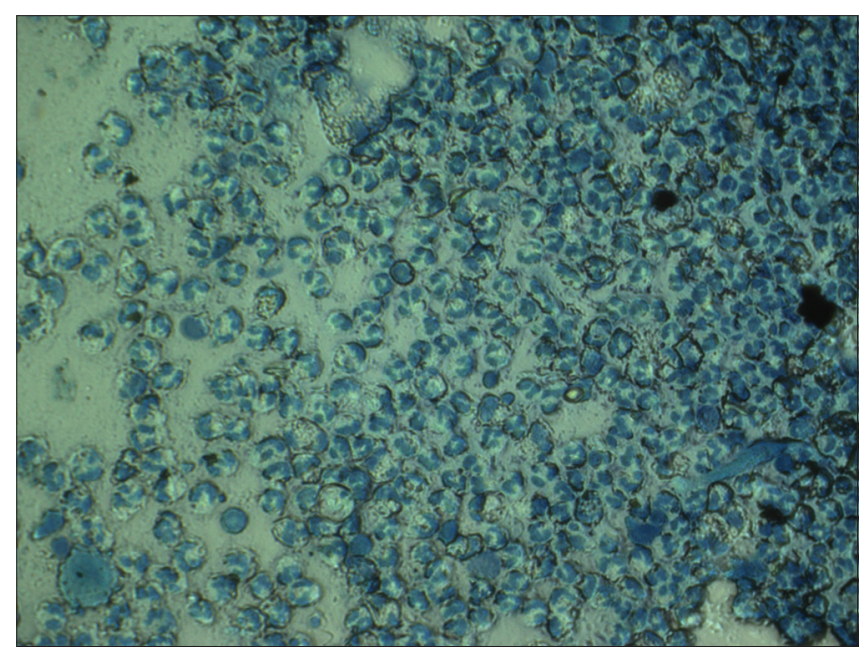

Figure 2: Microphotograph of the grain obtained during examination stained with methylene blue. No signs of actinomyces filiae.

but no signs of filaments typical for actinomycosis. A second sample was sent to the laboratory to perform aerobic and anaerobic cultivations - after two weeks, both turned out to be negative. In spite of the serious clinical condition of deep inflammatory cysts with concomitant discomfort, the authors decided to introduce a low dose of oral prednisone $(30 \mathrm{mg} /$ day) along with oral Amoxiciline/clavulanate compound $(2 \mathrm{xl} \mathrm{g} / \mathrm{day})$. The latter was discontinued after two weeks when negative bacteriological tests arrived. Topically, authors prescribed a preparation liquid containing chloramphenicol and resorcine, $3.5 \%$ of each in a $60 \%$ ethanol base twice a day. On a visit after two weeks, the inflammatory cysts appeared to be much smaller (less than half of initial status) but still persisted (Fig. 3). But most importantly,

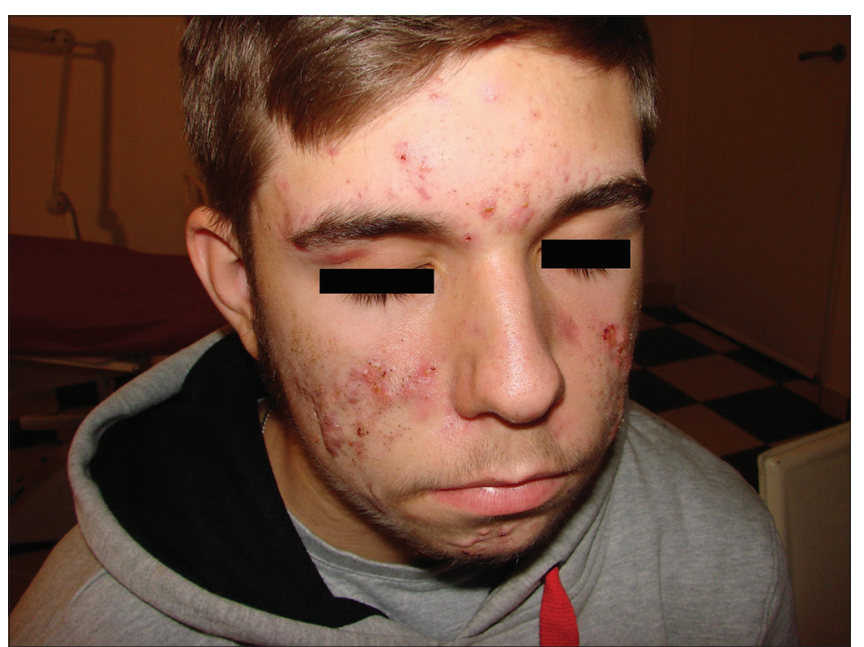

Figure 3: Clinical status before treatment. Please note large cyst on the right cheek.

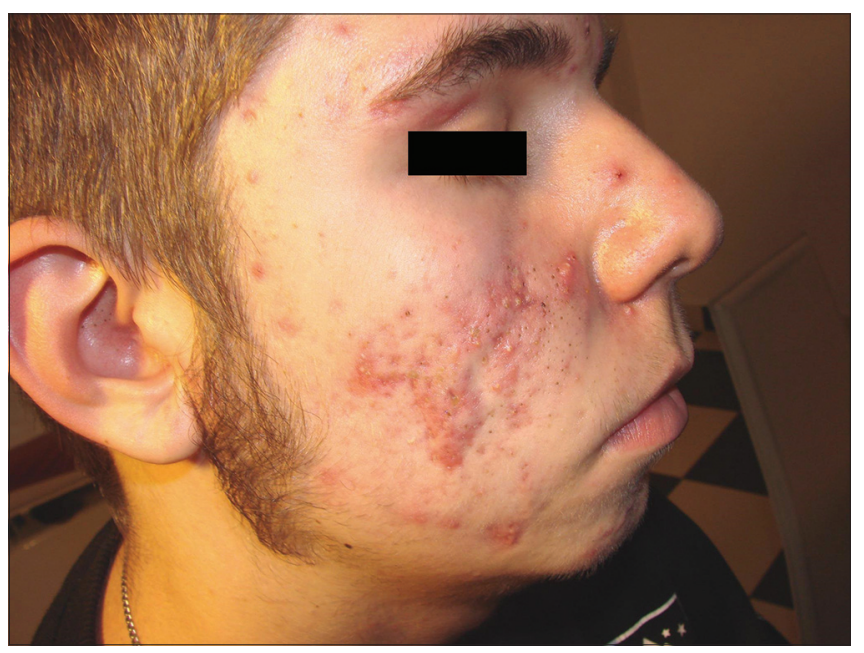

Figure 4: Clinical status during the treatment course showing partial improvement.

the patient did not report discomfort related with the disease anymore. The authors decided to slowly withdraw the prednisone during the next four weeks. After the prednisone was withdrawn, the patient was administered oral nabumetone (non - steroid anti inflammatory agent) in a dose of $1 \mathrm{~g}$ per day; topical treatment remained unchanged. Nabumetone was administered for another eight weeks. During this time, the large cysts have been disappearing, but slowly. At this point, the authors added to the existing topical treatment a prepared ointment containing cinnabar and sulfur (Rp. Mercury Sulfide 0.3, Precipitated Sulfur 4,0, Vaseline 10.0, Zinc Paste ad 30,0). During another four weeks of treatment, almost all the inflamed tumors disappeared, leaving numerous scars and crusts (Fig. 4). Patient remains under observation. 


\section{DISSCUSION}

Acne vulgaris is one of the most common skin diseases [5] and one of the most frequent diagnoses for patients who visit a dermatologist [6]. It is a chronic inflammatory disorder of the pilosebaceous unit and is characterised by non-inflammatory (comedones) and inflammatory lesions (papules, pustules, and nodules). The pathogenesis of acne vulgaris is complex and includes features of follicular hyperkeratosis, seborrhoea, bacterial colonisation and inflammation [7]. The present study reveals one of the most severe kinds of acne - acne conglobate, which in a presence of deep inflammation and concomitant general symptoms (pain, fever), is often described as acne fulminans. It is difficult to say whether the case described was complicated by actinomycosis; indeed, some clinical dates suggested it, but in spite of numerous systemic antibiotics used prior to the microbiological examination, its negative result is not surprising. While the clinical picture obviously suggested acne, authors hypothesised that there might also be an actinomycosis as a complication or as a separate phenomenon, as it was previously reported [8]. For this reason, a microscopic examination of the "sulfur grain" obtained during examination, stained with methylene blue was performed at the first visit (Fig. 2). Nevertheless, the inflammatory nodules and cysts in acne vulgaris should be differentiated with cysts present in a cervicofacial actinomycotic infection. The authors are aware of the fact that oral prednisone is often being improperly or unnecessarily prescribed these days, but this acne case is one of those where the introduction of a small dose of systemic corticosteroids might be necessary.

It is Propionibacterium acne that seems to play an important role in the pathogenesis of acne vulgaris because eventually it can lead to the presence of inflammatory cysts. These can create an important therapeutic problem even after the eradication of bacteria. Propionibacterium acne is an anaerobic, gram-positive pathogen that colonises in sebaceous follicles. Its cultures supernatants contain lipases and have been shown to activate inflammatory receptors, such as toll-like receptor 2 [9]. Propionibacterium acnes induced chemotactic factors may play an important role in attracting neutrophils to the pilosebaceous unit - hence the presence of neutrophiles in cysts in our study. Consequent release of lysosomal enzymes by the neutrophils leads to the rupture of the follicular epithelium and inflammation [10]. Propionibacterium acnes induces the monocytes in acne lesions to produce high levels of IL-1 and tumor necrosis factor- $\alpha(\mathrm{TNF}-\alpha)$ [11]. This process will give rise to inflammatory lesions: pustules, papules, nodules and cysts like in the above mentioned case.

While the eradication of bacteria is relatively simple these days, the damage that it leaves behind is not. Therefore, the authors suggest in conclusion that Acne conglobata should be understood as a case of acne where, due to the presence of lysosomal enzymes in affected follicles, destruction leaves large cysts characterized by a very chronic course. The management of acne at this stage seems no longer to be anti - microbic. Systemic anti-inflammatory agents are often necessary here; either non-steroid or low dose corticosteroids.

\section{REFERENCES}

1. Wong VK, Turmezei TD, Weston VC. Actinomycosis. BMJ. 2011; 343:d6099.

2. Pulverer G, Schütt-Gerowitt H, Schaal KP. Human cervicofacial actinomycoses: Microbiological data for 1997 cases. Clin Infect Dis. 2003;37:490-7.

3. Könönen E, Wade WG. Actinomyces and related organisms in human infections. Clin Microbiol Rev. 2015;28:419-42.

4. Bennhoff DF. Actinomycosis: Diagnostic and therapeutic considerations and a review of 32 cases. Laryngoscope. 1984;94:1198-217.

5. Cordain L, Lindeberg S, Hurtado M, Hill K, Eaton SB, Brand-Miller J. Acne vulgaris: A disease of Western Civilization. Arch Dermatol. 2002;38:1584-90.

6. Alexis A. Acne in Patients With Skin of Color. J Drugs Dermatol. 2011;10:13-6.

7. Läuchli S. Acne vulgaris. Curr Probl Dermatol. 2011;42:140-6.

8. Wasyłyszyn T, Borowska K. A case of cervicofacial actinomycosis with literature review. Our Dermatol Online. 2016;7:451-2.

9. Jugeau S, Tenaud I, Knol AC, Jarrousse V, Quereux G, Khammari A, et al. Induction of toll-like receptors by Propionibacterium acnes. Br J Dermatol. 2005;153:1105-13.

10. Mouser PE, Baker BS, Seaton ED, Chu AC. Propionibacterium acnes-reactive T helper-1 cells in the skin of patients with acne vulgaris. J Invest Dermatol. 2003;121:1226-8.

11. Vowels BR, Yang S, Leyden JJ. Induction of proinflammatory cytokines by a soluble factor of Propionibacterium acnes: Implications for chronic inflammatory acne. Infect Immun. 1995;63:3158-65.

Copyright by Magdalena Piotrkowicz, et al. This is an open access article distributed under the terms of the Creative Commons Attribution License, which permits unrestricted use, distribution, and reproduction in any medium, provided the original author and source are credited.

Source of Support: Nil Conflict of Interest: None declared. 